\title{
SELECTED GAMES OF THE NINTH MICROCOMPUTER CHESS CHAMPIONSHIP
}

\author{
The Editors \\ The following is a selection of the most important games of the tournament. \\ Mephisto X - Rebel \\ 1. d4 d5 2. c4 c6 3. cd5 cd5 4. Nf3 Nf6 5. Nc3 Nc6 6. Bf4 Bf5 7. e3 e6 8. Bb5 Nd7 9. Qa4 Rc8 10. Bc6 Rc6 11. \\ Qa7 Qc8 12. Qa5 Ra6 13. Qc7 Qa8 14. 0-0 Bd3 15. Rfc1 Rc6 16. Nd5 ed5 17. Rc6 bc6 18. Ne5 Ne5 19. Qe5 \\ Be7 20. Qg7 Rf8 21. Bh6 Kd8 22. a3 Qa7 23. Rc1 Qb6 24. b4 Bg6 25. Qe5 Re8 26. Bg5 Kd7 27. Be7 Re7 28. \\ Qf6 Qa6 29. Rc3 Qa4 30. Qf3 h5 31. h3 Qa8 32. Rc5 Re4 33. b5 cb5 34. Qe2 Qb7 35. Rb5 Qc6 36. Qb2 Re8 \\ 37. Rc5 Qa8 38. Qb5 Kd6 39. Rd5 Qd5 40. Qe8 Be4 41. f3 Bd3 42. a4 Qg5 43. g4 hg4 44. hg4 Da5 45. Kf2 \\ Bc4 46. Kg3 Kc7 47. Qe7 Kc8 48. g5 Qa4 49. g6 fg6 50. Qf8 Kc7 51. Qg7 Kd6 52. Qg6 Be6 53. Qg5 Qa2 54. \\ e4 Kc7 55. Qc5 Kb7 56. Qe7 Kc8 57. Qe8 Kc7 58. d5 Bd7 59. Qe5 Kc8 60. Qc3 Kb7 61. Qb4 Kc8 62. Qf8 Kc7 \\ 63. Qc5 Kd8 64. Qb6 Ke7 65. Qd4 draw (adjudication)
}

\section{Pandix - Nightmare}

1. Nf3 d5 2. b3 c5 3. Bb2 f6 4. e3 e5 5. d4 cd4 6. ed4 Qa5 7. Bc3 Bb4 8. Bb4 Qb4 9. c3 Qd6 10. Bb5 Kf8 11. de5 fe5 12. 0-0 Bg4 13. Be2 Bf3 14. Bf3 Nf6 15. Be2 Nc6 16. Qd3 e4 17. Qe3 Ne5 18. Nd2 Qc7 19. c4 Qa5 20. Rfd1 Rc8 21. cd5 Qd5 22. Qa7 Rc2 23. Nc4 Nd3 24. Qa8 Ne8 25. Ne3 Qd4 26. Bd3 ed3 27. Nc2 Qe4 28. Ne1 d2 29. Rd2 Qe5 30. Rad1 b5 31. Qa5 Nf6 32. Rd8 Ne8 33. Nf3 Qc5 34. Re8 Ke8 35. Rd8 Kf7 36. Rh8 Qc1 37. Qe1 Qe1 38. Ne1 h6 39. Kf1 Ke6 40. Ke2 1-0

\section{Why Not 89 - Mephisto X}

1. e4 d6 2. d4 Nf6 3. Nc3 c6 4. Nf3 Bg4 5. h3 Bh5 6. Be2 e6 7. Bf4 d5 8. 0-0 Ne4 9. Ne4 de4 10. Ne5 Be2 11. Qe2 Qd4 12. Rfd1 Qc5 13. Rd8 Kd8 14. Nf7 Ke8 15. Nh8 Qf5 16. Bd2 Bc5 17. Bc3 e5 18. b4 Bb6 19. Qc4 Bf2 20. Kh1 Kd7 21. Rd1 Kc7 22. Qg8 Bd4 23. Qg7 Nd7 24. Bd4 ed4 25. Rd4 Re8 26. Qf7 Qf7 27. Nf7 e3 28. Rd1 e2 29. Re1 Rf8 30. Re2 Rf7 31. a3 b5 32. Kg1 Nb6 33. Re4 a6 34. Rd4 Nc4 35. Rd3 Rd7 36. Rf3 Kb6 37. Kf2 Rd2 38. Kg3 Rc2 39. Kh2 Ra2 40. Rf8 Ra3 0-1

\section{Rebel - AI Chess}

1. d4 Nf6 2. c4 g6 3. Nc3 Bg7 4. e4 d6 5. Nf3 0-0 6. Be2 e5 7. de5 de5 8. Qd8 Rd8 9. Bg5 Re8 10. Nd5 Nd5 11. cd5 f6 12. Be3 f5 13. Rc1 fe4 14. Ng5 h6 15. Ne4 Bf5 16. f3 Rc8 17. 0-0 Nd7 18. g4 Be4 19. fe4 g5 20. Rc4 Nf6 21. Rfc1 Ne8 22. R4c2 Rd8 23. Bb5 Nf6 24. Rc7 Ne4 25. Bd7 b6 26. Bc6 Rab8 27. Ra7 Nf6 28. Rb7 Rb7 29. Bb7 b5 30. h3 Kf7 31. Rd1 Ke7 32. Bc5 Kf7 33. Bc6 Bf8 34. b4 Bd6 35. a3 e4 36. Kf2 Kg6 37. Bd4 Bf4 38. Bf6 Kf6 39. Bb5 Bc7 40. Ke2 Ke5 41. Bc6 Rf8 42. a4 Bd6 43. b5 Rf3 44. a5 Rh3 45. Rf1 Ra3 46. Rf5 Kd4 47. Rf6 Ra2 48. Kf1 Be5 49. Re6 Ra5 50. d6 Rg1 51. Ke2 e3 52. Bf3 Ra2 53. Ke1 Bg3 54. Kd1 Bf4 55. d7 Rd2 56. Ke1 Bg3 57. Kf1 Rf2 58. Kg1 Bh2 59. Kh1 Bc7 60. b6 Rf1 61. Kg2 Rf2 62. Kg1 Bd8 63. b7 Rb2 64. Re8 Rb1 65. Kg2 Rb2 66. Kf1 Rb1 67. Ke2 1-0

\section{AI Chess - Why Not 89}

1. c4 e6 2. d4 Nf6 3. Nf3 d5 4. e3 c5 5. Nc3 Nc6 6. a3 cd4 7. ed4 Be7 8. Bf4 0-0 9. Be2 dc4 10. Bc4 Nh5 11. Be3 Bd7 12. d5 Na5 13. Bd3 Bf6 14. Nd4 g6 15. de6 fe6 16. 0-0 Be5 17. Nf3 Bc3 18. bc3 Bc6 19. Nd4 Qd5 20. Qg4 Ng7 21. Bh6 Rae8 22. Rfe1 Bd7 23. Be4 Qc5 24. Qg3 Rc8 25. Ne2 Bb5 26. Nf4 Rfe8 27. Ng6 Qc7 28. Ne5 Rf8 29. Rad1 Qe7 30. Qh3 Qc7 31. Nd7 Bd7 32. Bg7 1-0

\section{Kempelen Atari - Mephisto X}

1. e4 c5 2. Nf3 d6 3. d4 cd4 4. Nd4 Nf6 5. Nc3 a6 6. Bg5 e6 7. f4 Be7 8. Be2 0-0 9. 0-0 h6 10. Bh4 Ne4 11. Be7 Nc3 12. Bd8 Nd1 13. Be7 Re8 14. Bd6 Nb2 15. Rab1 Na4 16. Bf3 Rd8 17. Bb8 Rd4 18. Be5 Rc4 19. Rfd1 Nc3 20. Rd8 Kh7 21. Rb3 Na2 22. Rd4 Rd4 23. Bd4 a5 24. c3 a4 25. Rb2 Nc1 26. Rb5 a3 27. Rb1 a2 28. Rc1 a1=Q 29. Ra1 Ra1 30. Kf2 g5 31. fg5 hg5 32. Bf6 Kg6 33. Bd4 Ra5 34. Kg3 e5 35. Be3 f5 36. Kf2 g4 37. Be2 Ra2 38. Bc5 b6 39. cb6 Ba6 40. Bc7 Re2 41. Kg1 f4 42. g3 Re1 43. Kg2 f3 44. Kf2 Re2 45. Kf1 f2 46. Be5 Rc2 47. c4 Bc4 48. Kg2 f1=Q mate 0-1 


\section{Rebel - Why Not 89}

1. d4 Nf6 2. c4 e6 3. Nf3 d5 4. Bg5 Be7 5. e3 0-0 6. Nc3 h6 7. Bh4 b6 8. Rc1 Bb7 9. Bf6 Bf6 10. cd5 ed5 11. b4 c5 12. bc5 bc5 13. dc5 Nd7 14. Nb5 Rc8 15. Nd6 Rc5 16. Nb7 Rc1 17. Qc1 Qb6 18. Bd3 Qb4 19. Nd2 Qb7 20. 0-0 Rc8 21. Qb1 Qb1 22. Rb1 Rc3 23. Rb3 d4 24. ed4 Rc1 25. Bf1 Rc2 26. Ne4 Ra2 27. Rb7 Nb6 28. Rb8 Kh7 29. Nf6 gf6 30. g3 Rd2 31. Rd8 Kg6 32. Ba6 Kf5 33. Kg2 Ke6 34. Re8 Kd6 35. Rh8 Rd4 36. Rh6 Ke7 37. h4 Ra4 38. Bf1 Rb4 39. Rh5 Kf8 40. Bd3 Rd4 41. Bc2 Rd2 42. Be4 Nc4 43. Rc5 Nb2 44. Ra5 Nd1 45. Ra7 Nf2 46. Bb1 Ng4 47. Kf3 Nh2 48. Ke4 Re2 49. Kd4 Rg2 50. Ba2 Kg7 51. Rf7 Kg6 draw

\section{Pandix - AI Chess}

1. Nf3 Nf6 2. b3 g6 3. Bb2 Bg7 4. e3 b6 5. Be2 Bb7 6. c4 c5 7. d4 0-0 8. 0-0 e6 9. Nd2 Nc6 10. a4 d5 11. dc5 bc5 12. cd5 Qd5 13. Re1 Nb4 14. Be5 Rd8 15. Qc1 Nd3 16. Bd3 Qd3 17. Qb2 Nh5 18. Bg7 Ng7 19. Rac1 Rab8 20. e4 Qd6 21. Qc3 Rbc8 22. h3 Nh5 23. e5 Qd3 24. Re3 Qc3 25. Rc3 Nf4 26. Rc4 Nd3 27. Rc3 Nb4 28. Rc4 Kg7 29. Ne4 Rd1 30. Re1 Rcd8 31. Nc5 Bf3 32. Rd1 Rd1 33. Kh2 Nc6 34. gf3 Ne5 35. Rc3 a5 36. Kg3 h5 37. f4 Nd7 38. Nd7 Rd7 39. Kf3 Kf6 40. Ke4 Rd2 41. Ke3 Rb2 42. Kf3 Ke7 43. Rd3 Rb1 44. Rc3 Kd6 45. Ke4 Rb2 46. Ke3 Kd5 47. h4 Rb1 48. f3 Kd6 49. Kf2 Rh1 50. Kg3 Rg1 51. Kf2 Rb1 52. Kg2 Ra1 53. Kh3 Ra2 54. Re3 Rb2 55. Rc3 Rf2 56. Kg3 Rd2 57. Rc8 Rd5 58. Ra8 Kc6 59. Rc8 Kb7 60. Re8 Rd7 61. Rf8 Kc6 draw (adjudicated)

\section{Kempelen Atari - Paul}

1. e4 g6 2. Nc3 Bg7 3. Bc4 d6 4. Nf3 Nf6 5. 0-0 0-0 6. d4 Ne4 7. Ne4 d5 8. Bd3 de4 9. Be4 c5 10. dc5 Qc7 11. Be3 Bb2 12. Rab1 Bg7 13. Qd3 Nc6 14. Rfd1 Rfd8 15. Qc4 Be6 16. Qa4 Bd7 17. Qb3 Rab8 18. Ng5 e6 19. Qc4 Na5 20. Qb4 h6 21. Nf3 f5 22. Bd3 e5 23. Bd2 Nc6 24. Bc4 Kh7 25. Qb3 e4 26. Ne1 Nd4 27. Qa3 Be5 28. Qh3 f4 29. Qa3 f3 30. gf3 Bh2 31. Kg2 ef3 32. Kh1 Bf5 33. Qe3 g5 34. Bd3 Rd5 35. c4 Bf4 36. cd5 Be3 37. Be3 Bd3 38. Rd3 Nf5 39. Nf3 Ne3 40. fe3 Qc5 41. Rb3 g4 42. Nd2 Qe2 43. e4 Qa2 44. Ra3 Qc2 45. Rc3 Qd1 46. Kg2 Rc8 47. Rc8 Qe2 48. Kg1 Qd3 49. Nc4 Qe4 50. d6 b5 51. Nd2 Qe3 52. Kf1 Qd2 53. Rc7 Kg6 54. Rc8 Qd6 55. Rg8 Kf5 56. Kf2 Qd4 57. Ke1 Qa1 58. Kd2 Q22 59. Ke3 Qg8 60. Kd3 g3 0-1

\section{Paul - Pandix}

1. g3 Nf6 2. Bg2 e6 3. Nf3 d5 4. 0-0 Nbd7 5. d3 Be7 6. Nc3 c5 7. Bf4 Qa5 8. e4 d4 9. Ne2 Nh5 10. Bd2 Qc7 11. c3 e5 12. cd4 cd4 13. Rc1 Qb6 14. Bh3 Qb2 15. g4 Nf6 16. g5 Nh5 17. bg4 g6 18. Bd7 Bd7 19. Ne5 Bh3 20. Nc4 Qb5 21. Rb1 Qc5 22. Qa4 Bd7 23. Qb4 b6 24. Qc5 Bc5 25. Ng3 Ng3 26. fg3 Be6 27. Rbc1 a6 28. Rf3 0-0 29. h4 b5 30. Ne5 Bd6 31. Nc6 Rfc8 32. Nd4 Rc1 33. Bc1 Ba2 34. Kf2 b4 35. Ke3 a5 36. Ne2 a4 37. d4 Bf8 38. Bf4 a3 39. d5 b3 40 Rf1 b2 41. Bb2 ab2 42. Nc3 Bg7 43. e5 Ra3 0-1

\section{Pandix - Rebel}

1. Nf3 d5 2. b3 Nc6 3. Bb2 Bg4 4. d4 e6 5. Nbd2 Nf6 6. g3 Bf5 7. Nh4 Ne4 8. Nf5 ef5 9. c3 Bd6 10. Bh3 Qg5 11. e3 0-0 12. 0-0 Ne7 13. Bg2 Rae8 14. c4 c6 15. Qc2 Ng6 16. Bc3 Qe7 17. Rad1 b6 18. a4 a6 19. Qd3 Ra8 20. Rfe1 Rfe8 21. Qc2 Rad8 22. Qd3 dc4 23. bc4 Nc3 24. Qc3 Bb4 25. Qb2 c5 26. d5 Ne5 27. Qb3 Ng4 28. Re2 Nf6 29. Nf3 Ne4 30. Rc2 Qf6 31. Ne1 Nc3 32. Rdc1 Ne4 33. Nd3 Ba5 34. Qa2 Qe7 35. Qa1 Qd6 36 Nf4 Re7 37. Rd1 Rde8 38. Qb2 Qd7 39. Nh5 f6 40. Qb3 Bb4 41. Rcc1 Nd2 42. Qc2 Ne4 43. Rd3 Qd6 44. Nf4 Ba5 45. Rcd1 Ng5 46. Rb3 g6 47. h4 Nf7 48. Rbb1 Ne5 49. h5 Bb4 50. Rbc1 Ba3 51. Ra1 Bb4 52. Ne6 Ng4 53. Rab1 Kh8 54. hg6 hg6 55. Ra1 Rh7 56. Rac1 Ba3 57. Rb1 Bb4 58. Ra1 Ree7 59. Rac1 Rh2 60. Nf4 Rh6 61. $\mathrm{Rb} 1 \mathrm{Kg} 80.5-0.5$ by adjudication

In the evening of September 13, the best players of Portorož challenged the partıcıpants of the microcomputer tournament to a 10-board match. The microcomputer team was supplemented by a Fidelity Mach 3 computer to round the number of games played to an even 10. All players and partıcipants were welcomed by the President of the Portorož chess club, Mr. Ivan Zabukoveć, who himself played in the match. The rate of play was 40 moves in 1 hour per side. After that, the players had to complete the game using an additional 30 minutes. The Presıdent of the Portorož chess club supervised the drawıng for colours with the Portorož fırst board, Mr. Veber, drawing the white pieces. The match was exciting to the end with the computers finally narrowly winning with 6 out of 10 points. The parrings for the match were: 


\begin{tabular}{|l|l|l|l|}
\hline 1. & Bojan Veber & Mephisto X & $0-1$ \\
2. & Evald Ule & A.I. Chess & $0.5-0.5$ \\
3. & Radovan Golja & Rebel & $0-1$ \\
4. & Petar Vasić & Quickstep & $0-1$ \\
5. & Andrej Znidarcić & Fidelity & $0-1$ \\
6. & Goran Treursić & Pandix & $0.5-0.5$ \\
7. & Ivan Zabukoveć & Why Not 89 & $0-1$ \\
8. & Franci Bodnjaveć & Paul & $1-0$ \\
9. & Roman Brecević & Kempelen Atari & $1-0$ \\
10. & Alex Murko & Nightmare & $1-0$ \\
\hline
\end{tabular}

From these games we selected:

\section{Bojan Veber - Mephisto X}

1. e4 d6 2. d4 Nf6 3. Nc3 c6 4. f4 Qa5 5. Bd3 e5 6. Nf3 Bg4 7. de5 de5 8. h3 Bf3 9. Qf3 Nbd7 10. g4 Bb4 11. Bd2 ef4 12. Qf4 Qe5 13. 0-0 0-0 14. Rad1 Qf4 15. Bf4 Bc3 16. bc3 h6 17. g5 hg5 18. Bg5 Rae8 19. Rf5 Re6 20. h4 b5 21. Re1 Rfe8 22. Ref1 Re5 23. R5f4 Rc5 24. Kg2 Rc3 25. Rf5 Ra3 26. h5 Ra2 27. h6 Ne5 28. hg7 Nd3 29. Bf6 Rc2 30. Kg3 Re4 31. Rh5 Re3 32. Kg4 Rc4 33. Kg5 Rg3 34. Kf5 Rc5 35. Ke4 0-1

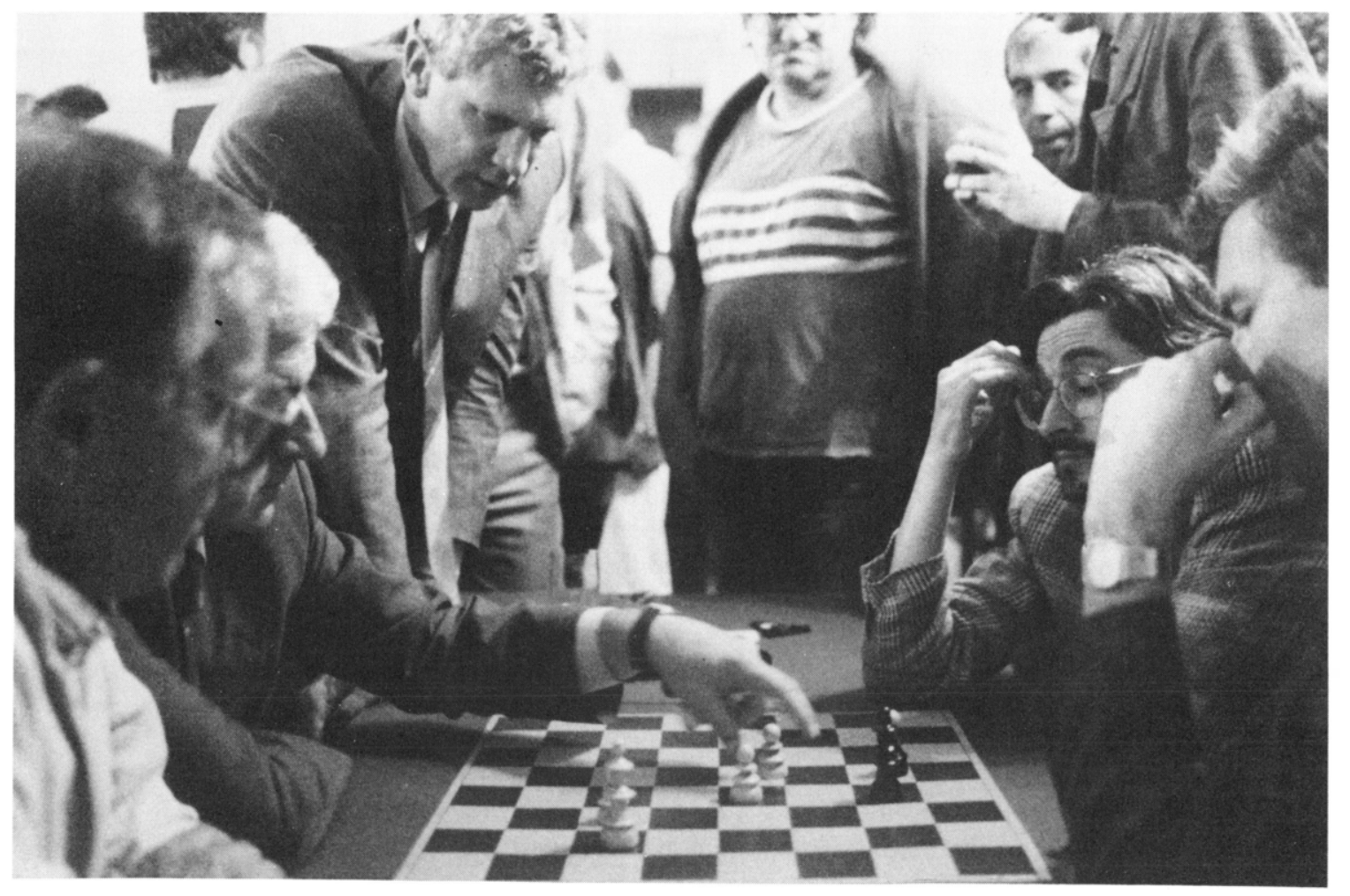

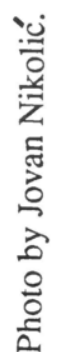

PUSHING PEOPLE WATCHING PUSHING PAWNS.

IGM Bruno Parma adjudicating the game Mephisto X versus Rebel as drawn. (Portorož, 1989) 